\title{
The Origin of Epithelium with Low-Grade Atypia in Early Gastric Cancer
}

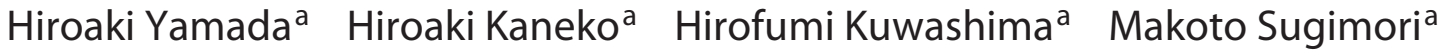 \\ Sho Tsuyuki ${ }^{a}$ Katsuyuki Sanga $^{a}$ Kuniyasu Irie $^{a}$ Tomohiko Sasaki $^{\mathrm{a}}$ \\ Masaaki Kondo $^{a}$ Akio Miyake ${ }^{b}$ Shin Maeda ${ }^{a}$ \\ aDepartment of Gastroenterology, Yokohama City University, Yokohama, Japan; 'bivision of Pathological Diagnosis, \\ Yokohama City University Hospital, Yokohama, Japan
}

\section{Keywords}

Epithelium with low-grade atypia - Helicobacter pylori .

Eradication - Gastric cancer

\begin{abstract}
Introduction: Helicobacter pylori (HP) infection causes chronic inflammation and atrophy of the gastric mucosa and thus a high risk of gastric cancer (GC). With the increasing success of HP infection treatment, a larger number of GCs that develop after eradication can be assessed. Several studies have shown that epithelium with low-grade atypia (ELA) is a frequent characteristic of these GCs, but the origin of this condition is unknown. In this study, we compared the mucin phenotype, cellular proliferation, and p53 staining in ELA and cancerous tissues obtained from patients with GC with and without HP eradication. Methods: The study population consisted of 23 patients with GC that developed after successful HP eradication therapy (eradicated group) and 24 patients with GC and HP infection (infected group). The prevalence of ELA was determined by hematoxylin and eosin staining. Tumor tissue and ELA samples were further analyzed by immunohistochemical staining for Muc5AC, Muc2, p53, and Ki-67. Results: The ELA coverage rate was significantly higher in the eradicated group than in the infected
\end{abstract}

Karger@karger.com www.karger.com/dig

Karger $\stackrel{\text { ' }}{5}$

GOPEN ACCESS
(C) 2022 The Author(s)

Published by S. Karger AG, Basel

This is an Open Access article licensed under the Creative Commons Attribution-NonCommercial-4.0 International License (CC BY-NC) (http://www.karger.com/Services/OpenAccessLicense), applicable to the online version of the article only. Usage and distribution for commercial purposes requires written permission. group. Gastric-type mucin was frequently expressed by the ELA, and the mucin phenotypes of ELA and cancerous areas differed in $75 \%$ of cases. The Ki- 67 labeling index was consistently lower in ELA than in the cancerous mucosa. Fourteen of 21 (66.7\%) cancerous lesions, but only 3 ELA samples, were p53-positive. Conclusion: In most cases, ELA on the surfaces of GCs seems to have originated from normal gastric cells, not from cancer cells.

(C) 2022 The Author(s)

Published by S. Karger AG, Basel

\section{Introduction}

Gastric cancer (GC) is among the most common cancers and accounts for nearly 750,000 deaths annually, making it the third leading cause of cancer death worldwide [1]. Helicobacter pylori (HP) infection is a major risk factor of GC $[2,3]$ as the chronic inflammation caused by the bacterium contributes to carcinogenesis [4]. Conversely, HP eradication has been shown to suppress GC development [5-9].

In 2013, the Japanese national health insurance system became the first in the world to cover HP eradication therapy for patients with chronic gastritis, and the number of successful eradication cases has increased steadily. 
At present, 1.5 million courses of eradication therapy have been administered in Japan [10]. The annual risk of GC development after HP eradication is reported to be $0.24 \%$ [11]. An understanding of the characteristics of GC discovered after eradication is important for the development of therapeutic strategies.

GC that develops after eradication tends to be difficult to diagnose by endoscopy because of its indistinct borders and lack of obviously malignant characteristics $[12,13]$. A possibly distinguishing feature is the greater frequency of epithelium with low-grade atypia (ELA) in patients with GC in whom HP infection has been eradicated successfully than in those with persisting infection $[12,14,15]$. A previous study showed that ELA derives from cancerous tissue as its genetic alterations are similar to those of tumor tissue [16]. However, that study was based on a small number of cases, and the researchers did not propose a mechanism by which cancer cells became low-grade atypical cells. Thus, whether ELA originates from cancerous or normal gastric tissue remains unclear.

In this study, we analyzed the ELA of patients with GC with and without HP eradication. Indicators of cellular proliferation and p53 staining demonstrated that ELA was largely noncancerous in origin.

\section{Patients and Methods}

\section{Patients}

From 164 early GC lesions detected by endoscopic submucosal dissection at our hospital between April 2015 and October 2018, poorly differentiated adenocarcinomas (signet ring-cell carcinomas, $n=3$; mixed differentiated and poorly differentiated adenocarcinomas, $n=5)$, cases lacking HP eradication information $(n=$ 77 ), and lesions that developed within 1 year after HP eradication $(n=32)$ were excluded. The remaining lesions were divided into two groups: those detected and diagnosed $>1$ year after successful HP eradication $(n=23)$ and those from patients with HP infection $(n=24)$. Successful eradication was confirmed by the urea breath test, with a cut-off level of 2.5\%. HP infection was confirmed by at least one of the following methods: (1) urea breath test, (2) HP stool antigen test, (3) serum immunoglobulin $\mathrm{G}$ antibody test, and (4) biopsy culture without eradication history.

This study was approved by the Institutional Review Board of the Faculty of Medicine, Yokohama City University (B160201008). All procedures were in accordance with the Declaration of Helsinki of 1964 and later versions. Informed consent was obtained from all patients prior to the endoscopic submucosal dissection.

Histopathological Assessment

ELA was defined as nonatypical epithelial cells: (1) present on the surface of GC tissue, (2) in columnar epithelium with spindleshaped or oval nuclei, (3) exhibiting nuclear polarity, and (4) form- ing a clear boundary with the cancerous lesion. Two physicians histologically assessed representative hematoxylin and eosinstained sections taken from the tumors at their largest diameters from the two groups, classifying them according to the extent of ELA as grades 0 (covering $0 \%$ of the entire cancer area), 1 (covering $<5 \%$ of the entire cancer area), 2 (covering $\geq 5 \%$ and $<30 \%$ of the entire cancer area), and 3 (covering $\geq 30 \%$ of the entire cancer area). The criteria about the ELA grade were based on the system used in the previous report [15].

\section{Immunohistochemical Assessment}

Representative sections taken from the tumors at their largest diameters and containing areas of ELA were immunohistochemically stained. After deparaffinization and rehydration, the slides were incubated with $3 \% \mathrm{H}_{2} \mathrm{O}_{2}$ at room temperature to block endogenous peroxidase activity. For antigen retrieval, the slides were incubated for $5 \mathrm{~min}$ at $110^{\circ} \mathrm{C}$ in an autoclave and subsequently incubated overnight at $4^{\circ} \mathrm{C}$ with the indicated primary antibodies. Biotinylated anti-rabbit, anti-rat, and anti-mouse secondary antibodies (1:200 dilution; Vector Laboratories, Burlingame, CA, USA) were next added for $30 \mathrm{~min}$ at room temperature. The solutions in the VECTASTAIN ABC Kit (Vector Laboratories) diluted 1:200 were applied to the slides. After $30 \mathrm{~min}$, the slides were developed with 3,3' -diaminobenzidine substrate (Muto Pure Chemicals, Tokyo, Japan) and counterstained with hematoxylin.

The primary antibodies used in this study were mouse anti-p53 (clone DO-7; Dako Japan, Tokyo, Japan), anti-Ki-67 (Dako Japan), anti-mucin (MUC)5AC (Novocastra Laboratories, Newcastle, UK), and anti-MUC2 (Dako Japan) monoclonal antibodies. The Ki-67 labeling index (LI) was determined by counting at least 200 gastric epithelial cells in each sample. MUC5AC, MUC2, and p53 positivity was defined as staining of $>10 \%$ of the section. MUC types were classified as gastric (MUC5AC+/MUC2-), intestinal (MUC2+/MUC5AC-), and mixed (MUC5AC+/MUC2+). MUC expression, the Ki-67 LI, and p53 expression were determined in the cancerous tissues and ELA from the 47 patients.

\section{Statistical Analysis}

All statistical analyses were performed using $\mathrm{R}$ version 3.3.1. Differences in ELA grade were assessed using Fisher's exact test, and differences in average Ki-67 LIs were assessed using the paired $t$ test. $p$ values $<0.05$ were considered to indicate statistical significance.

\section{Results}

\section{Histological Grades of ELA in the Eradicated and} Infected Groups

A representative image of ELA, showing the columnar epithelium and spindle-shaped and oval nuclei, is presented as Figure 1. Lesion samples were obtained from the 23 patients in the eradicated group (GC diagnosed $>1$ year after successful HP eradication) and 24 patients in the infected group. In the eradicated group, the median period from the eradication was 67 months (range 17115 months). The median tumor size in the infected 

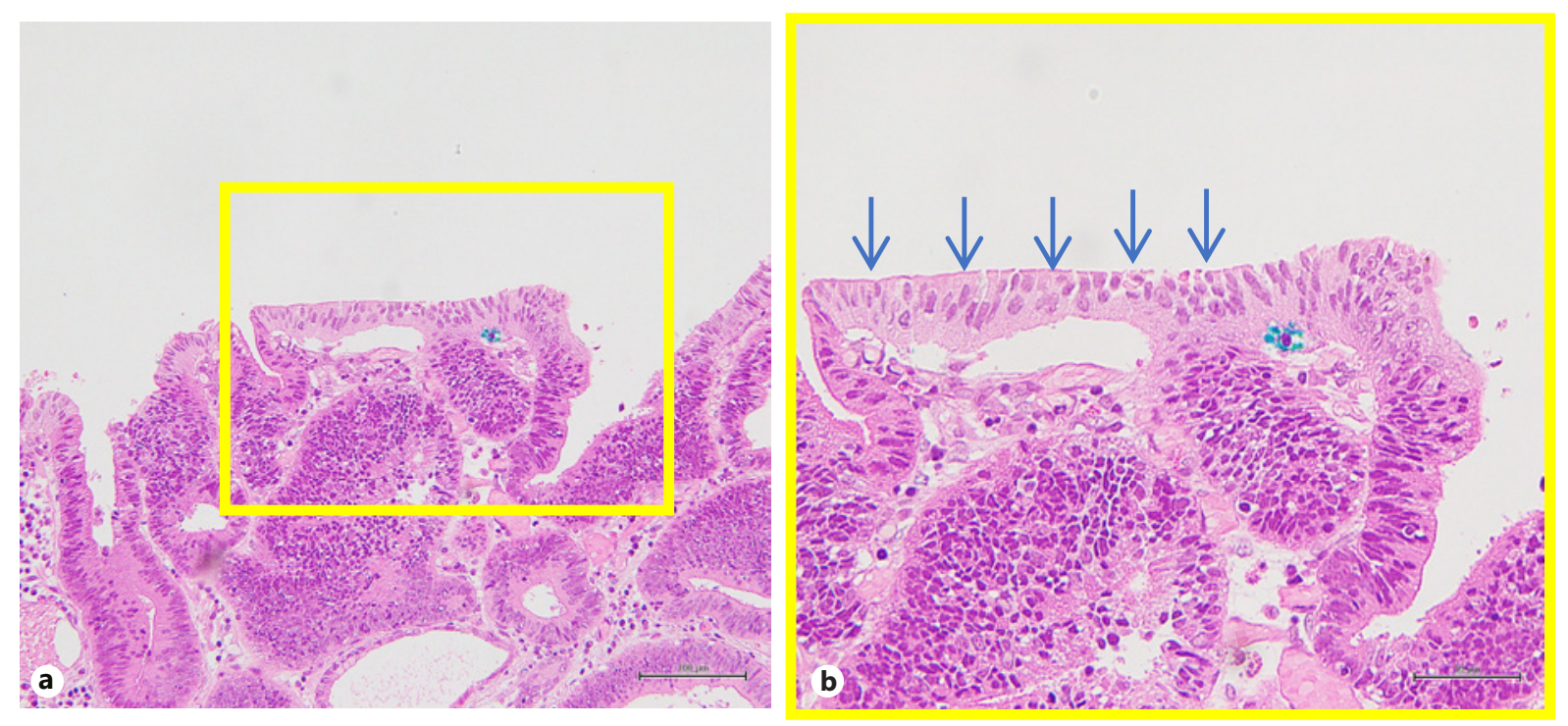

Fig. 1. Microscopic features of cancerous tissue and ELA from patients with GC after HP eradication. a Representative image of ELA (yellow box), $\times 100$. b Magnified image of the area shown in the yellow box in $\mathbf{a}, \times 200$.

group was $12 \mathrm{~mm}$ (range, 5-66 mm), and the median tumor size in the eradicated group was $12 \mathrm{~mm}$ (range, 4-45 $\mathrm{mm})$. There was no significant difference between the two groups.

The histological grades of the lesions in the two groups are summarized in Table 1 . Grade 0 (covering $0 \%$ of the entire cancer area) was significantly more frequent in the infected group than in the eradicated group ( $p=0.008$ ), whereas grade 3 (covering $\geq 30 \%$ of the entire cancer area) was significantly more frequent in the eradicated group than in the infected group $(p<0.001)$. These results suggest that ELA is found more frequently in GC developing after HP eradication than in that developing against a background of active infection, as reported previously [15]. There was no significant correlation between the term after the eradication and the ELA grade $(r=0.47)$. In addition, there was also no significant relevance between the tumor size and the ELA grade $(r=0.079)$ (online suppl. Fig. S1; see www.karger. com/doi/10.1159/000521875 for all online suppl. material).

\section{MUC Phenotypes of ELA-Positive GC}

Tumor phenotypes are generally determined according to the expression of MUC markers, including MUC2, a marker of intestinal goblet cells (intestinal type) and
Table 1. Histological grading of ELA in GCs from patients with eradicated HP and continued infection

\begin{tabular}{llll}
\hline & $\begin{array}{l}\text { Infected group } \\
\text { (24 lesions) }\end{array}$ & $\begin{array}{l}\text { Eradicated group } \\
\text { (23 lesions) }\end{array}$ & $p$ value \\
\hline Grade 0 & 18 & 8 & 0.008 \\
Grade 1 & 4 & 2 & \\
Grade 2 & 2 & 4 & $<0.001$ \\
Grade 3 & 0 & 9 & \\
\hline
\end{tabular}

MUC5AC, a marker of surface gastric epithelium (gastric type). MUC expression in the ELA and cancerous mucosa of the 20 ELA-positive cases was analyzed by immunohistochemistry (no tissue sample from one HP-infected ELA-positive patient was available for analysis). The MUC phenotypes of cancerous tissues were gastric $(n=6)$, intestinal $(n=8)$, and mixed $(n=6)$. Of the 6 lesions with gastric MUC phenotypes, the MUC phenotypes of the ELA were gastric in 4 cases and intestinal in 2 cases. Of the 6 lesions with mixed MUC phenotypes, the MUC phenotypes of the ELA were gastric in 5 cases and mixed in 1 case. Of the 8 lesions with intestinal MUC phenotypes, the MUC phenotypes of the ELA were gastric in 5 cases and mixed in 3 cases (Fig. 2). The typical 
Fig. 2. Mucin phenotypes of ELA-positive GCs in the 47 study patients. G, gastric; I, intestinal; GI, mixed gastric and intestinal.

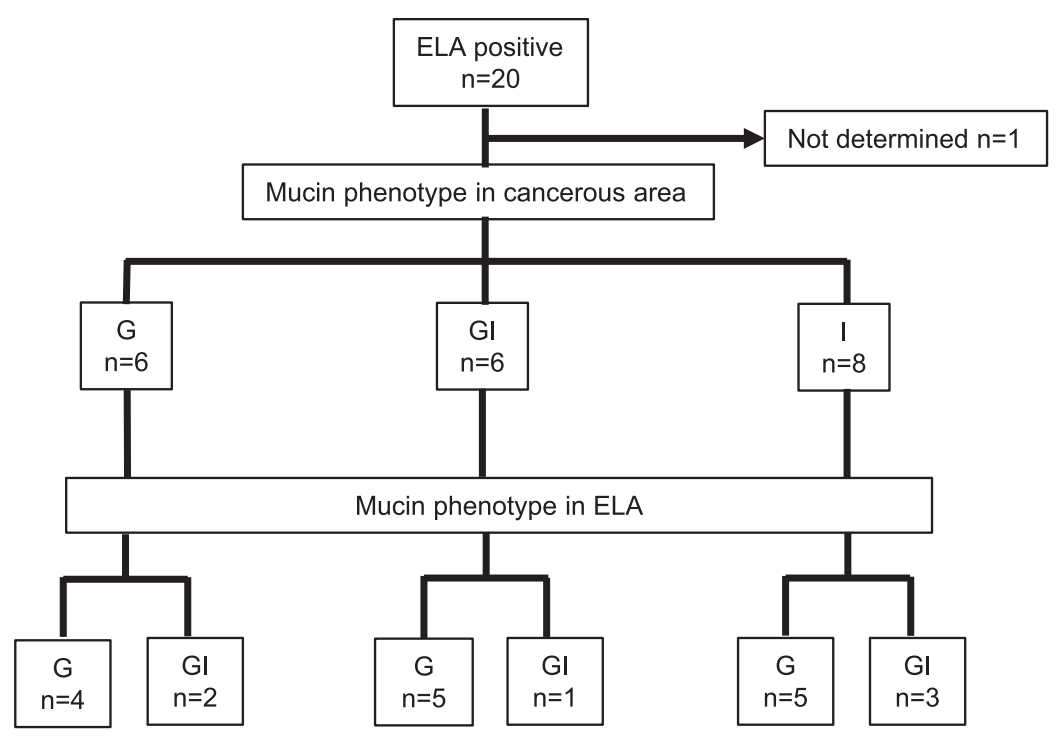

G: gastric type I: intestinal type GI: mixed type
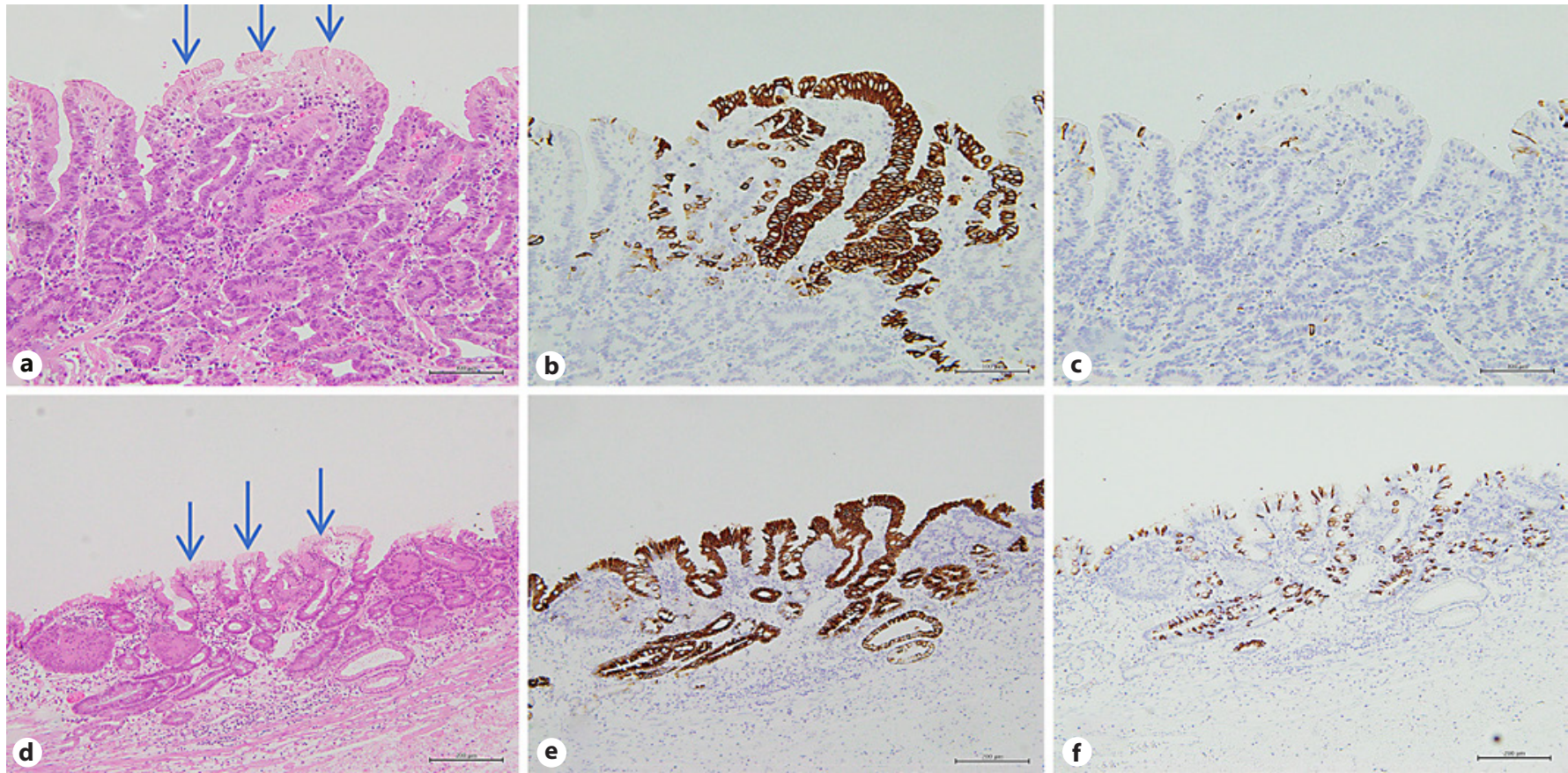

Fig. 3. Microscopic features and mucin expression of ELA from GCs that developed after HP eradication. a H\&E staining of ELA (arrows). Immunohistochemical staining indicating MUC5AC positivity (b) and MUC2 negativity (c). These findings are consistent with a gastric mucin phenotype. H\&E staining of ELA (d), MUC5AC positivity (e) and MUC2 positivity (f). These findings are consistent with the mixed phenotype. a-c, $\times 200 ; \mathbf{d}-\mathbf{f}$, $\times 100$. 


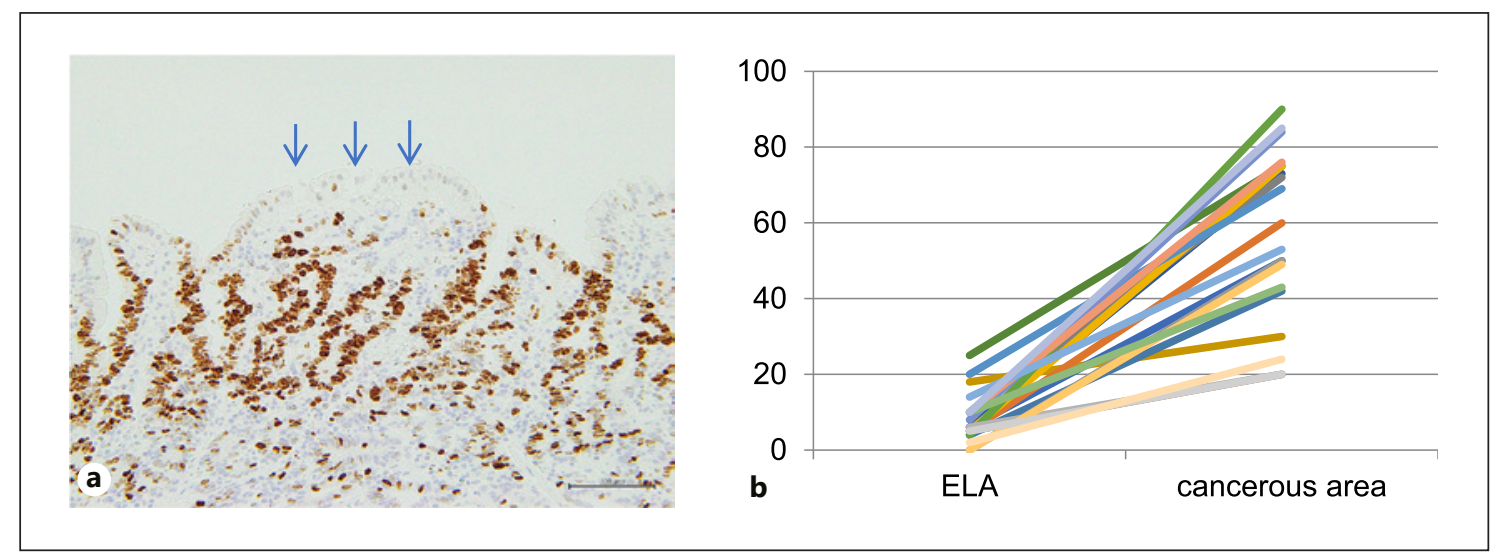

Fig. 4. Microscopic features of Ki-67 expression in GC. Immunohistochemical Ki-67 staining of ELA in GC tissue (arrows) (a), Ki-67 labeling indices for ELA and cancerous tissues from patients with ELA-positive GC (b).

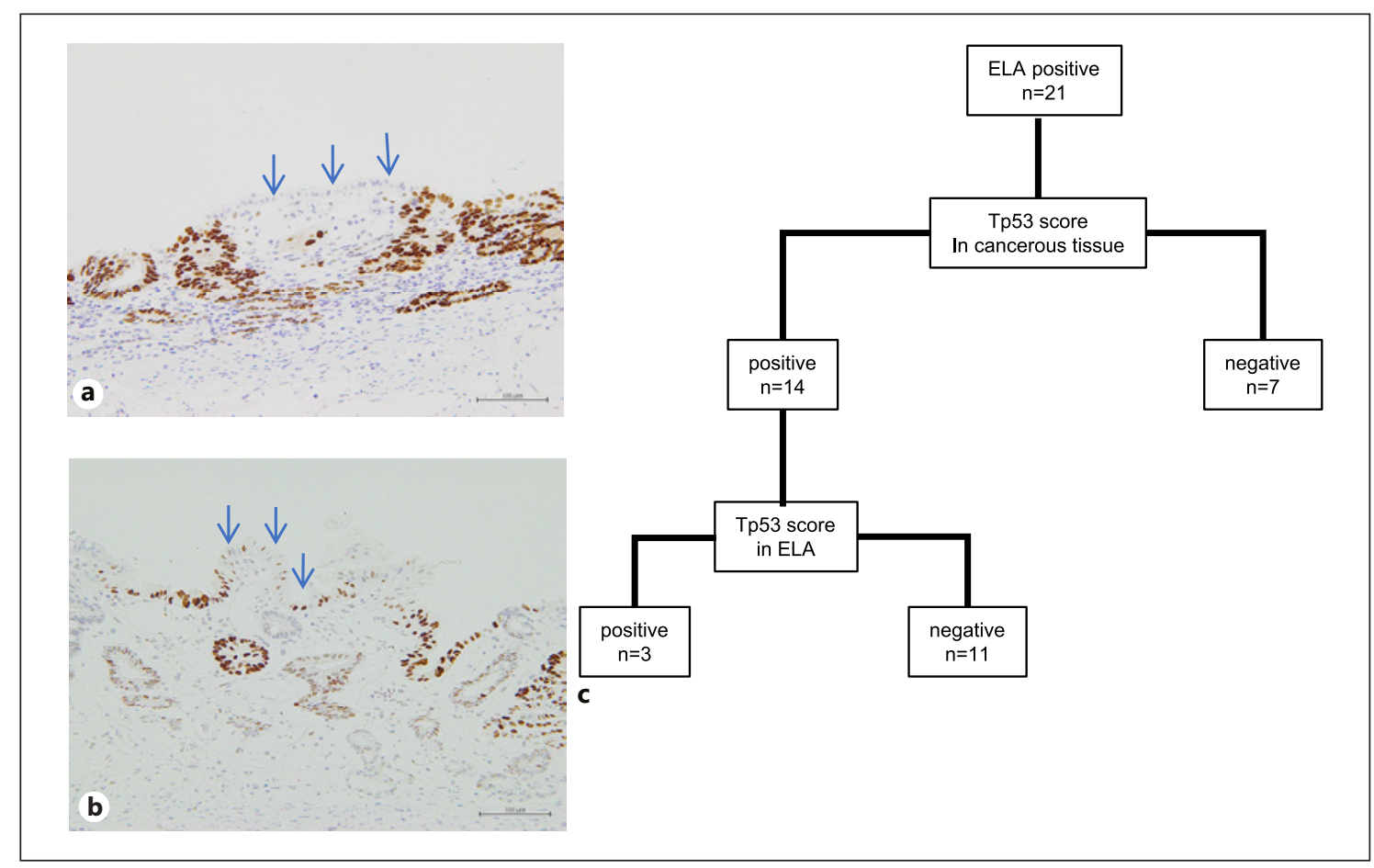

Fig. 5. Microscopic features of p53 expression in GC. a, b Immunohistochemical p53 staining of ELA in GC tissue (arrows). Immunohistochemical staining indicating p53 negativity (a) and p53 positivity (b). c Flowchart showing p53 expression in cancerous tissues and ELA.

features of gastric- and mixed-type ELA are shown in Figure 3. All ELA samples were positive for MUC5AC, a gastric mucin. In 15 of 20 (75\%) cases, the MUC phenotypes differed between ELA and cancerous tissues, indicating that the ELA did not originate from the cancerous lesions.

Epithelium with Low-Grade Atypia in Early Gastric Cancer

\section{Ki-67 Immunohistochemistry}

$\mathrm{Ki}-67$ is a nuclear protein expressed during the cell cycle, and its expression correlates with the tumor cell proliferation rate. In the present sample, the average Ki-67 LI was lower in ELA than in cancerous tissue (8.238 \pm 6.433 vs. $55.238 \pm 23.134, p<0.001$, Fig. 4 ). In most cases, the 
Ki-67 LI was similar to that of the surrounding noncancerous area (data not shown). These results also suggest that the ELA was derived from the noncancerous area, not the cancerous tissue.

\section{p53 Immunohistochemistry}

Mutations in p53 result in the production of stable p53 proteins, which can be detected by immunohistochemistry and serve as cancer markers. In this study, immunohistochemical analysis revealed p53 positivity in 14 of 21 (66.7\%) ELA-positive cancerous tissue samples (Fig. 5). Of these cases, $11(78.6 \%)$ ELA samples were p53 negative. These results provide further evidence that is derived primarily ELA from noncancerous tissue.

\section{Discussion}

The aim of this study was to determine whether ELA originates from cancerous or noncancerous tissue through the immunohistochemical examination of MUC2, MUC5AC, and p53 expression and the Ki-67 LI in ELA-positive cancer tissues. In all cases, ELA was characterized by gastric-type MUC (MUC5AC), as reported previously [15], suggesting that MUC5AC expression is a defining characteristic of ELA. The MUC phenotype was altered in $75 \%$ of cancerous tissues, possibly reflecting MUC5AC production by cells of different origin. Alternatively, the cells may have had the same origin but then differentiated, acquiring different MUC phenotypes. The transcription factor Sox 2 controls the gastric phenotype and is transcribed in gastric-type and gastric/intestinal mixed-type epithelial cells, in accordance with MUC5AC expression, whereas Cdx 1 and $\mathrm{Cdx} 2$ are upregulated together with MUC2 in intestinaltype and gastric/intestinal mixed-type epithelial cells [17]. Thus, the differentiation of gastric- and intestinaltype cells according to transcription factor expression has been proposed $[17,18]$. In the case of HP infection, the bacterium or the inflammatory response to it may change epithelial-cell MUC expression from the gastric to the intestinal type via the expression of Cdx2. This change would occur mainly in stem cells or cells at similar levels of differentiation but not in fully differentiated cells [19]. These observations indicate that ELA does not originate from cancerous lesions, in accordance with our finding of ELA positivity in 75\% of the GC tissues examined in this study.

The number of Ki-67-positive cells (Ki-67 LI) was clearly lower in ELA than in cancerous tissue in this study.
In samples from the eradicated group, the Ki-67 LI of ELA was nearly the same as that of the background gastric mucosa, suggesting a shared feature of the two tissues. However, even in cancerous lesions without ELA, the Ki67 LI decreased with increasing differentiation, such that Ki-67 was a less informative marker than MUC.

Mutations in p53 are found in $50-70 \%$ of early GCs $[20,21]$. In our study, p53 nuclear staining was detected in $67 \%$ of the cancer tissues and not detected in $79 \%$ (11 of 14) of the ELA samples, suggesting that ELA did not derive from cancer in those cases. By contrast, p53 nuclear staining was observed in $21 \%$ ( 3 of 14) of the ELA samples, but the number of p53-positive cells was much smaller than that in the cancerous tissues. In those cases, p53 nuclear staining may not have been caused by a p53 mutation but instead by normal p53 activation and translocation.

The Ki-67 LI and p53 immunohistochemistry is often used in the diagnosis of colitis-associated cancer (CAC), which, while clearly different from GC, shares features related to inflammation-associated carcinogenesis [22]. In the pathological diagnosis of CAC, the distinction of CAC lesions from nonneoplastic inflammatory changes can be difficult. In such cases, p53 and Ki-67 immunohistochemistry may be useful as a secondary tool. The application of this strategy to the diagnosis of ELA in GC may enable the identification of the very small number of cases in which ELA is derived from cancerous tissue.

It is very difficult to analyze the mechanism how ELA was generated from normal gastric mucosa. We hypothesize that ELA may be one of the regenerating atypia. This situation arises when the gastric mucosa is reacting to regeneration after erosion or ulceration [23]. It is likely that eradication of HP increases the gastric acid output [24], which may lead to surface injury of the tumor lesion and induce regenerative changes. However, precise mechanism is still unknown and should be analyzed in the future.

This study has several limitations, the most important of which is the small number of patients included. Additional limitations are the retrospective design, which may entail selection bias and the single-center setting. Larger, prospective studies involving multiple centers are needed to confirm our findings.

In summary, we demonstrated that ELA on the surfaces of GC tumors derives from normal mucosa. Elucidation of the characteristics of ELA will lead to a better understanding of early-stage GC. 


\section{Statement of Ethics}

This study was approved by the Institutional Review Board of the Faculty of Medicine, Yokohama City University (B160201008). All procedures were in accordance with the Declaration of Helsinki of 1964 and later versions. Written informed consent was obtained from all patients before they were included in the study.

\section{Conflict of Interest Statement} close.

The authors declare that they have no conflict of interest to dis-

\section{Funding Sources}

No funding was received regarding this study.

\section{Author Contributions}

H.Y. and S.M. drafted the manuscript. H.K., H.K., M.S., S.T., K.S., K.I., T.S., M.K., and A.M. revised the manuscript critically for important intellectual content. S.M. supervised this review article. All the authors were involved in study design and interpretation. All the authors agree to be accountable for all aspects of the work in ensuring that questions related to the accuracy or integrity of any part of the work are appropriately investigated and resolved.

\section{Data Availability Statement}

All data generated or analyzed during this study are included in this article. Further inquiries can be directed to the corresponding author.

\section{References}

1 Ferlay J, Colombet M, Soerjomataram I, Mathers C, Parkin DM, Pineros M, et al. Estimating the global cancer incidence and mortality in 2018: GLOBOCAN sources and methods. Int J Cancer. 2019;144(8):1941-53.

2 Uemura N, Okamoto S, Yamamoto S, Matsumura N, Yamaguchi S, Yamakido M, et al. Helicobacter pylori infection and the development of gastric cancer. N Engl J Med. 2001; 345(11):784-9.

3 Huang JQ, Sridhar S, Chen Y, Hunt RH. Meta-analysis of the relationship between Helicobacter pylori seropositivity and gastric cancer. Gastroenterology. 1998;114(6):1169-79.

4 Schistosomes, liver flukes and, Helicobacter pylori. IARC Working Group on the evaluation of carcinogenic risks to humans, Lyon, 7-14 June 1994. IARC Monogr Eval Carcinog Risks Hum. 1994;61:1-241.

5 Fukase K, Kato M, Kikuchi S, Inoue K, Uemura N, Okamoto S, et al. Effect of eradication of Helicobacter pylori on incidence of metachronous gastric carcinoma after endoscopic resection of early gastric cancer: an open-label, randomised controlled trial. Lancet. 2008;372(9636):392-7.

6 Take S, Mizuno M, Ishiki K, Hamada F, Yoshida T, Yokota K, et al. Seventeen-year effects of eradicating Helicobacter pylori on the prevention of gastric cancer in patients with peptic ulcer; a Prospective Cohort Study. J Gastroenterol. 2015;50(6):638-44

7 Yoon SB, Park JM, Lim CH, Cho YK, Choi MG. Effect of Helicobacter pylori eradication on metachronous gastric cancer after endoscopic resection of gastric tumors: a metaanalysis. Helicobacter. 2014;19(4):243-8.

8 Lee YC, Chiang TH, Chou CK, Tu YK, Liao WC, Wu MS, et al. Association between Helicobacter pylori eradication and gastric cancer incidence: a systematic review and meta-analysis. Gastroenterology. 2016;150(5):1113-24. e5.

9 Choi IJ, Kook MC, Kim YI, Cho SJ, Lee JY, Kim CG, et al. Helicobacter pylori therapy for the prevention of metachronous gastric cancer. N Engl J Med. 2018;378(12):1085-95.

10 Tsuda M, Asaka M, Kato M, Matsushima R, Fujimori K, Akino K, et al. Effect on Helicobacter pylori eradication therapy against gastric cancer in Japan. Helicobacter. 2017;22: e12415.

11 Kamada T, Hata J, Sugiu K, Kusunoki H, Ito $\mathrm{M}$, et al. Clinical features of gastric cancer discovered after successful eradication of Helicobacter pylori: results from a 9-Year Prospective Follow-Up Study in Japan. Aliment Pharmacol Ther. 2005;21:1121-6.

12 Saka A, Yagi K, Nimura S. Endoscopic and histological features of gastric cancers after successful Helicobacter pylori eradication therapy. Gastric Cancer. 2016;19(2):524-30.

13 Kobayashi M, Hashimoto S, Nishikura K, Mizuno K, Takeuchi M, Sato Y, et al. Magnifying narrow-band imaging of surface maturation in early differentiated-type gastric cancers after Helicobacter pylori eradication. J Gastroenterol. 2013;48(12):1332-42.

14 Ito M, Tanaka S, Takata S, Oka S, Imagawa S, Ueda $\mathrm{H}$, et al. Morphological changes in human gastric tumours after eradication therapy of Helicobacter pylori in a short-term followup. Aliment pharmacol Ther. 2005;21(5): 559-66.

15 Kitamura Y, Ito M, Matsuo T, Boda T, Oka S, Yoshihara M, et al. Characteristic epithelium with low-grade atypia appears on the surface of gastric cancer after successful Helicobacter pylori eradication therapy. Helicobacter. 2014;19(4):289-95.
16 Masuda K, Urabe Y, Ito M, Ono A, Nelson $\mathrm{H}$, Nakamura K, et al. Genomic landscape of epithelium with low-grade atypia on gastric cancer after Helicobacter pylori eradiation therapy. J Gastroenterol. 2019;54:907-15.

17 Camilo V, Garrido M, Ricardo S, Amaral AL Barros R, Chaves P, et al. Differentiation reprogramming in gastric intestinal metaplasia and dysplasia: role of SOX2 and CDX2. Histopathology. 2015;66(3):343-50.

18 Tatematsu M, Tsukamoto T, Inada K. Stem cells and gastric cancer: role of gastric and intestinal mixed intestinal metaplasia. Cancer Sci. 2003;94(2):135-41.

19 Shibata W, Sue S, Tsumura S, Ishii Y, Sato T, Kameta E, et al. Helicobacter-induced gastric inflammation alters the properties of gastric tissue stem/progenitor cells. BMC Gastroenterol. 2017;17(1):145.

20 Fenoglio-Preiser CM, Wang J, Stemmermann GN, Noffsinger A. TP53 and gastric carcinoma: a review. Hum Mutat. 2003;21(3):25870.

21 Cancer Genome Atlas Research Network. Comprehensive molecular characterization of gastric adenocarcinoma. Nature. 2014 Sep 11;513(7517):202-9.

22 Kawachi H. Histopathological diagnosis of ulcerative colitis-associated neoplasia. Dig Endosc. 2019 Apr;31(Suppl 1):31-5.

23 Rugge M, Correa P, Dixon MF, Hattori T, Leandro $\mathrm{G}$, Lewin $\mathrm{K}$, et al. Gastric dysplasia: the Padova international classification. Am J Surg Pathol. 2000;24(2):167-76.

24 Haruma K, Mihara M, Okamoto E, Kusunoki H, Hananori M, Tanaka S, et al. Eradication of Helicobacter pylori increases gastric acidity in patients with atrophic gastritis of the corpus: evaluation of 24-h $\mathrm{pH}$ monitoring. Aliment Pharmacol Ther. 1999;13(2):155-62. 